\title{
Selections of set-valued functions satisfying the general linear inclusion
}

\author{
Andrzej Smajdor and Joanna Szczawińska
}

\begin{abstract}
Applying the classical Banach fixed point theorem we prove that a set-valued function satisfying a general linear functional inclusion admits a unique selection fulfilling the corresponding functional equation. We also adopt the method of the proof for investigating the Rassias stability of general linear equation.
\end{abstract}

Mathematics Subject Classification. 54C65, 54C60, 39B82.

Keywords. Set-valued function, selection, stability.

\section{Introduction}

Let $(Y,\|\cdot\|)$ be a real normed space. We denote by $n(Y)$ the family of all nonempty subsets of $Y$ and by $\operatorname{cl}(Y), \operatorname{ccl}(Y), \mathrm{c}(Y)$ and $\operatorname{cp}(Y)$ we denote collections of all closed, convex closed, compact and complete members of $n(Y)$, respectively.

The number

$$
\operatorname{diam} A:=\sup \{\|a-b\|: a, b \in A\}
$$

is said to be the diameter of $A \in n(Y)$. We say that a set-valued function $F: K \rightarrow n(Y)$ (an "s.v. function" for abbreviation) is with bounded diameter if the function $K \ni x \mapsto \operatorname{diam} F(x) \in \mathbb{R}$ is bounded.

In [5], the authors proved that if $S$ is a commutative semigroup with zero and $(Y,\|\cdot\|)$ is a real Banach space, then $F: S \rightarrow \operatorname{ccl}(Y)$ is a subadditive s.v. function; i.e.,

$$
F(x+y) \subset F(x)+F(y), \quad x, y \in S,
$$

with bounded diameter admits a unique additive selection. Popa in [11] proved that if $\emptyset \neq K$ is a convex cone in a real vector space $X$ (i.e., $t_{1} K+t_{2} K \subset K$ for all $\left.t_{1}, t_{2} \geq 0\right)$ and $F: K \rightarrow \operatorname{ccl}(Y)$ (where $(Y,\|\cdot\|)$ is a real Banach space) is an s.v. function with bounded diameter fulfilling the inclusion

$$
F(\alpha x+\beta y) \subset \alpha F(x)+\beta F(y), \quad x, y \in K,
$$

for $\alpha, \beta>0, \alpha+\beta \neq 1$, then there exists exactly one additive selection of $F$. 
Nikodem and Popa in [9] and Piszczek in [10] proved the following theorem.

Theorem 1.1. Let $K$ be a convex cone in a real vector space $X,(Y,\|\cdot\|)$ a real Banach space and $\alpha, \beta, p, q>0$. Consider an s.v. function $F: K \rightarrow \operatorname{ccl}(Y)$ with bounded diameter fulfilling the inclusion

$$
\alpha F(x)+\beta F(y) \subset F(p x+q y), \quad x, y \in K .
$$

If $\alpha+\beta<1$, then there exists a unique selection $f: K \rightarrow Y$ of $F$ satisfying the equation

$$
\alpha f(x)+\beta f(y)=f(p x+q y), \quad x, y \in K .
$$

If $\alpha+\beta>1$, then $F$ is single valued.

The case of $p+q=1$ was investigated by Popa in [13], Inoan and Popa in [7] and recently, by Smajdor and Szczawińska in [14].

In this paper, we determine the conditions for which an s.v. function $F: K \rightarrow n(Y)$ satisfying the inclusion

$$
\alpha F(x)+\beta F(y) \subset F(p x+q y), \quad x, y \in K,
$$

where $|\alpha|<p$ and $\operatorname{diam} F(x) \leq M\|x\|, x \in K$ (for some $M>0$ ) admits a selection satisfying the corresponding functional equation. It is easy to check that if $F$ satisfies the opposite inclusion, then it is single valued. Theorem 2.1 and also the method of its proof are used for the investigation of the Rassias stability of general linear functional equation.

\section{The main theorem}

Let $(X,\|\cdot\|)$ and $(Y,\|\cdot\|)$ be real normed spaces and let $K$ be a nonempty subset of $X$. Consider an s.v. function $F: K \rightarrow n(Y)$. A function $f: K \rightarrow Y$ is a selection of the s.v. function $F$ if and only if $f(x) \in F(x), x \in K$. Let

$$
\operatorname{Sel}(F):=\{f: K \rightarrow Y: f(x) \in F(x), x \in K\} .
$$

It is easy to check that if there exists a constant $M>0$ such that $\operatorname{diam} F(x) \leq$ $M\|x\|$ for all $x \in K$, then the function

$$
d(f, g):=\sup \left\{\frac{\|f(x)-g(x)\|}{\|x\|}, x \in K \backslash\{0\}\right\}, \quad f, g \in \operatorname{Sel}(F),
$$

is a metric in $\operatorname{Sel}(F)$. Moreover, if $F(x)$ is complete for every $x \in K$, the metric space $(\operatorname{Sel}(F), d)$ is complete. Obviously, the convergence in the space $(\operatorname{Sel}(F), d)$ implies the pointwise convergence on the set $K$.

Theorem 2.1. Let $(X,\|\cdot\|)$ and $(Y,\|\cdot\|)$ be real normed spaces and let $K \subset X$ be such that $0 \in K$. Assume that $p, q>0$ and $\alpha, \beta \in \mathbb{R}$ are fixed and one of the following conditions holds:

(i) $|\alpha|<p$ and $K \subset p K$,

(ii) $|\beta|<q$ and $K \subset q K$. 
Consider an s.v. function $F: K \rightarrow \operatorname{cp}(Y)$ such that $0 \in F(0)$ and

$$
\operatorname{diam} F(x) \leq M\|x\|, \quad x \in K,
$$

for some positive constant $M$. If

$$
\alpha F(x)+\beta F(y) \subset F(p x+q y), \quad x, y \in K, p x+q y \in K,
$$

then there exists a unique selection $f: K \rightarrow Y$ of $F$ such that

$$
\alpha f(x)+\beta f(y)=f(p x+q y), \quad x, y \in K, p x+q y \in K .
$$

Proof. Assume that $|\alpha|<p$ and $K \subset p K$. Since diam $F(0)=0$ and $0 \in F(0)$, $F(0)=\{0\}$. Putting $y=0$ in (2.1), we obtain

$$
\alpha F\left(\frac{x}{p}\right) \subset F(x), \quad x \in K .
$$

Let

$$
\mathcal{T}(g)(x):=\alpha g\left(\frac{x}{p}\right), \quad x \in K, g \in \operatorname{Sel}(G) .
$$

By $(2.2), \mathcal{T}(g) \in \operatorname{Sel}(F), g \in \operatorname{Sel}(F)$. Moreover, for every $g_{1}, g_{2} \in \operatorname{Sel}(F)$,

$$
\begin{aligned}
d\left(\mathcal{T}\left(g_{1}\right), \mathcal{T}\left(g_{2}\right)\right) & =|\alpha| \sup \left\{\frac{\left\|g_{1}\left(\frac{x}{p}\right)-g_{2}\left(\frac{x}{p}\right)\right\|}{\|x\|}, x \in K \backslash\{0\}\right\} \\
& =\frac{|\alpha|}{p} \sup \left\{\frac{\left\|g_{1}\left(\frac{x}{p}\right)-g_{2}\left(\frac{x}{p}\right)\right\|}{\left\|\frac{x}{p}\right\|}, x \in K \backslash\{0\}\right\} \\
& \leq \frac{|\alpha|}{p} d\left(g_{1}, g_{2}\right) .
\end{aligned}
$$

Since $|\alpha|<p$, the map $\mathcal{T}: \operatorname{Sel}(F) \rightarrow \operatorname{Sel}(F)$ is contractive in the complete metric space $(\operatorname{Sel}(F), d)$, so by the Banach theorem, it has a unique fixed point $f$ and $\lim _{n \rightarrow \infty} T^{n}(g)=f$ for each $g \in \operatorname{Sel}(F)$. Hence $f: K \rightarrow Y$ is the unique selection of the s.v. function $F$ such that

$$
f(x)=\alpha f\left(\frac{x}{p}\right), \quad x \in K .
$$

Fix $g \in \operatorname{Sel}(F)$ and $x, y \in K$ such that $p x+q y \in K$. Then

$$
\frac{x}{p}, \frac{y}{p}, \frac{p x+q y}{p} \in K .
$$

By (2.1),

$$
\alpha g\left(\frac{x}{p}\right)+\beta g\left(\frac{y}{p}\right), g\left(\frac{p x+q y}{p}\right) \in F\left(\frac{p x+q y}{p}\right) .
$$

Hence

$$
\begin{aligned}
\left\|\alpha g\left(\frac{x}{p}\right)+\beta g\left(\frac{y}{p}\right)-g\left(\frac{p x+q y}{p}\right)\right\| & \leq \operatorname{diam} F\left(\frac{p x+q y}{p}\right) \\
& \leq M\left\|\frac{p x+q y}{p}\right\| .
\end{aligned}
$$


Thus

$$
\|\alpha \mathcal{T}(g)(x)+\beta \mathcal{T}(g)(y)-\mathcal{T}(g)(p x+q y)\| \leq \frac{|\alpha|}{p} M\|p x+q y\|
$$

for every $x, y \in K$ such that $p x+q y \in K$. Proceeding by induction, we get

$$
\left\|\alpha \mathcal{T}^{n}(g)(x)+\beta \mathcal{T}^{n}(g)(y)-\mathcal{T}^{n}(g)(p x+q y)\right\| \leq\left(\frac{|\alpha|}{p}\right)^{n} M\|p x+q y\|
$$

for every $n \in \mathbb{N}$ and all $x, y \in K$ with $p x+q y \in K$. Letting $n \rightarrow \infty$, we obtain

$$
\alpha f(x)+\beta f(y)=f(p x+q y), \quad x, y \in K, p x+q y \in K .
$$

Let $X$ and $Y$ be real vector spaces and $K$ a convex cone in $X$. It is easy to check that if $f: K \rightarrow Y$ satisfies the equation

$$
\alpha f(x)+\beta f(y)=f(p x+q y), \quad x, y \in K,
$$

and $f(0)=0$, then $f$ is additive; i.e.,

$$
f(x)+f(y)=f(x+y), \quad x, y \in K .
$$

Corollary 2.2. Let $p, q>0$ and $|\alpha|<p$ (or $|\beta|<q)$. Assume that $(X,\|\cdot\|)$ and $(Y,\|\cdot\|)$ are normed spaces, $K$ a convex cone in $X$ and $F: K \rightarrow \operatorname{cp}(Y)$ an s.v. function such that $0 \in F(0)$ and

$$
\operatorname{diam} F(x) \leq M\|x\|, \quad x \in K,
$$

for some constant $M>0$. If

$$
\alpha F(x)+\beta F(y) \subset F(p x+q y), \quad x, y \in K,
$$

then the unique selection $f: K \rightarrow Y$ of $F$ fulfilling the equation

$$
\alpha f(x)+\beta f(y)=f(p x+q y), \quad x, y \in K,
$$

is additive.

Remark 2.3. If $|\alpha|=p$ and $|\beta|=q$, the above corollary is not true.

Proof. Let $K=[0,+\infty)$ and let $F: K \rightarrow \mathrm{c}(\mathbb{R})$ be an s.v. function defined by

$$
F(x)=[0, x], \quad x \in K,
$$

and $\operatorname{diam} F(x)=|x|, x \in K$. Then

$$
F(x+y)=F(x)+F(y), \quad x, y \in K,
$$

and for every $a \in[0,1]$, the function $f(x)=a x, x \in K$, is an additive selection of $F$. 


\section{Stability results}

In the first part of this section we present an application of Theorem 2.1 to the investigation of the Rassias stability of the general linear functional equation

$$
\alpha f(x)+\beta f(y)=f(p x+q y) .
$$

For definition and more results in the Rassias stability theory see, for instance, $[6,8]$. The general linear equation was considered by several authors (for example, $[1,2,12])$. A set-valued approach can be found in [3].

Gajda showed in [4] (see also [8, Theorem 2.6]) that for $\varepsilon>0$ there is a function $f: \mathbb{R} \rightarrow \mathbb{R}$ satisfying the inequality

$$
|f(x)+f(y)-f(x+y)| \leq \varepsilon(|x|+|y|), \quad x, y \in \mathbb{R},
$$

while there is no constant $M \geq 0$ and no additive function $f_{0}: \mathbb{R} \rightarrow \mathbb{R}$ such that

$$
\left|f(x)-f_{0}(x)\right| \leq M|x|, \quad x \in \mathbb{R} .
$$

We apply the method used in the proof of Theorem 2.1 to obtain the stability result for equation (3.1). We will denote by $\mathbb{R}_{+}$and $\mathbb{Q}_{+}$the set of all nonnegative real and rational numbers, respectively.

Theorem 3.1. Let $K$ be a convex cone in a real normed space $(X,\|\cdot\|)$, $(Y,\|\cdot\|)$ a real Banach space and $\varepsilon: \mathbb{R}_{+} \times \mathbb{R}_{+} \rightarrow \mathbb{R}_{+}$an $\mathbb{R}_{+}$-homogenous function. Assume that $p, q>0, \alpha, \beta \in \mathbb{R}$ are fixed and $|\alpha|<p$. If a function $f: K \rightarrow Y$ satisfies

$$
\|\alpha f(x)+\beta f(y)-f(p x+q y)\| \leq \varepsilon(\|x\|,\|y\|), \quad x, y \in K,
$$

then there exists a unique function $f_{0}: K \rightarrow Y$ fulfilling the functional equation

$$
\alpha f_{0}(x)+\beta f_{0}(y)=f_{0}(p x+q y), \quad x, y \in K,
$$

and such that

$$
\left\|f(x)-f_{0}(x)-f(0)\right\| \leq M\|x\|, \quad x \in K,
$$

for some $M>0$. Moreover,

$$
\left\|f(x)-f_{0}(x)-f(0)\right\| \leq \frac{\varepsilon(1,0)}{p-\alpha}\|x\|, \quad x \in K,
$$

and $f_{0}$ is an additive function. In particular, if $\varepsilon(1,0)=0$, then

$$
f(x)=f_{0}(x)-f(0), \quad x \in K .
$$

Proof. Let $g: K \rightarrow Y$ be given by $g(x)=f(x)-f(0), x \in K$. It is easy to check that $g(0)=0$ and

$$
\|\alpha g(x)+\beta g(y)-g(p x+q y)\| \leq \varepsilon(\|x\|,\|y\|), \quad x, y \in K .
$$

Hence, for all $x, y \in K$,

$$
\alpha g(x)+\beta g(y) \in g(p x+q y)+\varepsilon(\|x\|,\|y\|) B,
$$


where $B$ denotes the unit closed ball in the space $Y$. Setting $y=0$ and replacing $x$ by $x / p$ in (3.3), we obtain

$$
\alpha g\left(\frac{x}{p}\right) \in g(x)+\varepsilon\left(\frac{\|x\|}{p}, 0\right) B=g(x)+\frac{\varepsilon(1,0)}{p}\|x\| B, \quad x \in K .
$$

Consider an s.v. function $G: K \rightarrow \operatorname{cl}(Y)$ given by

$$
G(x)=g(x)+\frac{\varepsilon(1,0)}{p-\alpha}\|x\| B, \quad x \in K .
$$

Then

$$
\operatorname{diam} G(x)=\frac{2 \varepsilon(1,0)}{p-\alpha}\|x\|, \quad x \in K,
$$

and

$$
\begin{aligned}
\alpha G\left(\frac{x}{p}\right) & =\alpha g\left(\frac{x}{p}\right)+\alpha \frac{\varepsilon(1,0)}{p-\alpha}\left\|\frac{x}{p}\right\| B \\
& \subset g(x)+\frac{\varepsilon(1,0)}{p}\left(1+\frac{\alpha}{p-\alpha}\right)\|x\| B=G(x)
\end{aligned}
$$

for all $x \in K$. The idea of the proof is the same as before so we only give a sketch. The function $T: \operatorname{Sel}(G) \rightarrow \operatorname{Sel}(G)$, given by

$$
T(h)(x):=\alpha h\left(\frac{x}{p}\right), \quad x \in K, h \in \operatorname{Sel}(G),
$$

is contraction with the constant $|\alpha| / p$. By the Banach theorem, there exists a unique function $f_{0} \in \operatorname{Sel}(G)$ such that

$$
f_{0}(p x)=\alpha f_{0}(x), \quad x \in K,
$$

and $\lim _{n \rightarrow \infty} T^{n}(g)=f_{0}$. By the definition of $G$,

$$
\left\|f_{0}(x)-g(x)\right\| \leq \frac{\varepsilon(1,0)}{p-\alpha}\|x\|, \quad x \in K .
$$

Since $g$ satisfies (3.2) and $\varepsilon$ is positively homogeneous,

$$
\|\alpha T(g)(x)+\beta T(g)(y)-T(g)(p x+q y)\| \leq \frac{|\alpha|}{p} \varepsilon(\|x\|,\|y\|)
$$

for all $x, y \in K$. Proceeding by induction, we get

$$
\left\|\alpha T^{n}(g)(x)+\beta T^{n}(g)(y)-T^{n}(g)(p x+q y)\right\| \leq\left(\frac{|\alpha|}{p}\right)^{n} \varepsilon(\|x\|,\|y\|)
$$

for every $x, y \in K$ and $n \geq 1$. It follows that

$$
\alpha f_{0}(x)+\beta f_{0}(y)=f_{0}(p x+q y), \quad x, y \in K .
$$

Since $f_{0}(0)=0, f_{0}$ is additive.

For the end assume that $f_{1}: K \rightarrow Y$ is a solution of equation (3.1) such that

$$
\left\|f_{1}(x)-g(x)\right\| \leq M\|x\|, \quad x \in K,
$$


for some positive $M$. Then

$$
f_{1}(0)=0, \quad \alpha f_{1}\left(\frac{x}{p}\right)=f_{1}(x), \quad x \in K
$$

and

$$
\left\|f_{1}(x)-f_{0}(x)\right\| \leq\left(M+\frac{\varepsilon(1,0)}{p-\alpha}\right)\|x\|, \quad x \in K .
$$

It is easy to check that

$$
\left\|f_{1}(x)-f_{0}(x)\right\| \leq\left(\frac{|\alpha|}{p}\right)^{n}\left(M+\frac{\varepsilon(1,0)}{p-\alpha}\right)\|x\|, \quad x \in K, n \in \mathbb{N} .
$$

Hence $f_{1}=f_{0}$, which ends the proof.

In the second part of this section, we apply Theorem 2.1 to obtain a stability result for equation (3.1).

Theorem 3.2. Let $K$ be a convex cone in a real normed space $(X,\|\cdot\|),(Y,\|\cdot\|)$ a real Banach space and $p, q>0, \alpha, \beta \in \mathbb{R}$ fixed constants such that $|\alpha|<p$. Consider $\eta \geq 0$ and a function $f: K \rightarrow Y$ satisfying the inequality

$$
\|\alpha f(x)+\beta f(y)-f(p x+q y)\| \leq \eta(\|x\|+\|y\|), \quad x, y \in K .
$$

If there exists a constant $\lambda>0$ such that

$$
(1+\lambda \alpha)\|x\|+(1+\lambda \beta)\|y\| \leq \lambda\|p x+q y\|, \quad x, y \in K,
$$

then there exists a unique function $f_{0}: K \rightarrow Y$ fulfilling the functional equation

and such that

$$
\alpha f_{0}(x)+\beta f_{0}(y)=f_{0}(p x+q y), \quad x, y \in K,
$$

$$
\left\|f(x)-f_{0}(x)-f(0)\right\| \leq M\|x\|, \quad x \in K,
$$

for some $M>0$. Moreover,

$$
\left\|f(x)-f_{0}(x)-f(0)\right\| \leq \lambda \eta\|x\|, \quad x \in K,
$$

and $f_{0}$ is an additive function.

Proof. Let $g: K \rightarrow Y$ be given by $g(x)=f(x)-f(0), x \in K$. As in the proof of Theorem 3.1, $g(0)=0$ and

$$
\|\alpha g(x)+\beta g(y)-g(p x+q y)\| \leq \eta(\|x\|+\|y\|), \quad x, y \in K .
$$

In particular, for all $x, y \in K$,

$$
\alpha g(x)+\beta g(y) \in g(p x+q y)+\eta(\|x\|+\|y\|) B,
$$

where $B$ is the unit closed ball in $Y$. Define an s.v. function $G: K \rightarrow \operatorname{cl}(Y)$ as follows:

$$
G(x)=g(x)+\lambda \eta\|x\| B, \quad x \in K .
$$

Then $0 \in G(0), \operatorname{diam} G(x)=2 \eta \lambda\|x\|, x \in K$, and

$$
\alpha G(x)+\beta G(y) \subset G(p x+q y), \quad x, y \in K .
$$


Indeed, let $x, y \in K$ be fixed. By condition (3.4),

$$
\begin{aligned}
\alpha G(x)+\beta G(y) & =\alpha g(x)+\beta g(y)+\eta(\lambda \alpha\|x\|+\lambda \beta\|y\|) B \\
& \subset g(p x+q y)+\eta(\|x\|+\|y\|) B+\eta(\lambda \alpha\|x\|+\lambda \beta\|y\|) B \\
& \subset g(p x+q y)+\eta \lambda\|p x+q y\| B \\
& =G(p x+q y) .
\end{aligned}
$$

By Theorem 2.1, there exists exactly one selection $f_{0}: K \rightarrow Y$ of the s.v. function $G$ satisfying equation (3.1). Hence

$$
\left\|f(x)-f_{0}(x)-f(0)\right\| \leq \lambda \eta\|x\|, \quad x \in K .
$$

Since $f_{0}(0)=0, f_{0}$ must be additive. The proof that $f_{0}$ is the only solution of equation (3.1) such that

$$
\left\|f(x)-f_{0}(x)-f(0)\right\| \leq M\|x\|, \quad x \in K,
$$

for some $M>0$ runs as before.

Example. Let $|\alpha|<p$ and $|\beta|<q$. Every convex cone

$$
K \subset\left\{\left(x_{1}, \ldots, x_{n}\right) \in \mathbb{R}^{n}: x_{1} \geq 0 \wedge \cdots \wedge x_{n} \geq 0 \vee x_{1} \leq 0 \wedge \cdots \wedge x_{n} \leq 0\right\}
$$

satisfies condition (3.4) with

$$
\lambda \geq \max \left\{\frac{1}{p-\alpha}, \frac{1}{q-\beta}\right\}
$$

and with the norm in $\mathbb{R}^{n}$ given by

$$
\left\|\left(x_{1}, \ldots, x_{n}\right)\right\|=\left|x_{1}\right|+\cdots+\left|x_{n}\right|, \quad\left(x_{1}, \ldots, x_{n}\right) \in \mathbb{R}^{n} .
$$

\section{The selection theorem}

In this section we present a certain consequence of Theorem 2.1 for the case of a compact-valued s.v. function $F$ satisfying the inclusion (1.1) and such that the function $x \mapsto \operatorname{diam} F(x)$ maps bounded sets onto bounded ones.

Lemma 4.1. Let $p, q>0$ and $\alpha, \beta \in \mathbb{R}$ be fixed. Assume that $X$ and $Y$ are real vector spaces, $K$ a convex cone in $X$ and $\mathcal{K}$ a collection of nonempty subsets of $Y$ closed under intersections of chains. Let $F: K \rightarrow \mathcal{K}$ be an s.v. function fulfilling the inclusion (1.1) and $0 \in F(0)$. There exists a minimal s. $v$. function $F_{0}: K \rightarrow \mathcal{K}$ such that

(i) $F_{0}(x) \subset F(x), x \in K$,

(ii) $0 \in F_{0}(0)$,

(iii) $\alpha F_{0}(x)+\beta F_{0}(y) \subset F_{0}(p x+q y), x, y \in K$.

Proof. Define the set $\mathcal{F}$ of all s.v. functions $H: K \rightarrow \mathcal{K}$ such that

$$
H(x) \subset F(x), \quad x \in K,
$$

$0 \in H(0)$ and

$$
\alpha H(x)+\beta H(y) \subset H(p x+q y), \quad x, y \in K .
$$


The set $\mathcal{F}$ is nonempty, because $F \in \mathcal{F}$, and partially ordered by the relation

$$
H_{1} \preceq H_{2} \Longleftrightarrow H_{1}(x) \subset H_{2}(x), \quad x \in K, H_{1}, H_{2} \in \mathcal{F} .
$$

It is enough to prove that there is a minimal element in $\mathcal{F}$. Let $\mathcal{C}$ be a chain in $\mathcal{F}$. Put

$$
H_{0}(x)=\bigcap\{H(x): H \in \mathcal{C}\}, \quad x \in K .
$$

Since $\mathcal{K}$ is closed under intersections, $H_{0}(x) \in \mathcal{K}, x \in K$. Obviously, $0 \in$ $H_{0}(0)$ and $H_{0}(x) \subset H(x), x \in K$. Fix now $x, y \in K$. For every $H \in \mathcal{C}$,

$$
\alpha H_{0}(x)+\beta H_{0}(y) \subset \alpha H(x)+\beta H(y) \subset H(p x+q y),
$$

hence

$$
\alpha H_{0}(x)+\beta H_{0}(y) \subset H_{0}(p x+q y) .
$$

Therefore, the s.v. function $H_{0}: K \rightarrow \mathcal{K}$ belongs to $\mathcal{F}$ and $H_{0} \preceq H, H \in \mathcal{C}$. By the Kuratowski-Zorn lemma, there exists a minimal element $F_{0}$ in the set $\mathcal{F}$.

Lemma 4.2. Let $p, q>0$ and $\alpha, \beta \in \mathbb{R}$ be fixed. Assume that $X$ and $Y$ are real vector spaces, $K$ a convex cone in $X$ and $\mathcal{K}$ a family of nonempty subsets of $Y$ such that $\alpha \mathcal{K} \subset \mathcal{K}$ and $\lambda \mathcal{K} \subset \mathcal{K}, \lambda \in \mathbb{Q}_{+}$. Consider an s.v. function $F: K \rightarrow \mathcal{K}$. If $F_{0}: K \rightarrow \mathcal{K}$ is a minimal s.v. function such that

(i) $F_{0}(x) \subset F(x), x \in K$,

(ii) $0 \in F_{0}(0)$,

(iii) $\alpha F_{0}(x)+\beta F_{0}(y) \subset F_{0}(p x+q y), x, y \in K$,

then $F_{0}$ is superadditive and $\mathbb{Q}_{+}$-homogeneous; i.e.,

$$
F_{0}(x)+F_{0}(y) \subset F_{0}(x+y), \quad x, y \in K,
$$

and $F_{0}(\lambda x)=\lambda F_{0}(x), x \in K, \lambda \in \mathbb{Q}_{+}$.

Proof. Putting $y=0$ in (iii) and by (ii),

$$
\alpha F_{0}\left(\frac{x}{p}\right) \subset F_{0}(x), \quad x \in K .
$$

Define

$$
H(x)=\alpha F_{0}\left(\frac{x}{p}\right), \quad x \in K .
$$

Then $H(x) \in \mathcal{K}, H(x) \subset F_{0}(x) \subset F(x), x \in K$ and $0 \in \alpha F_{0}(0)=H(0)$. Observe that the s.v. function $H: K \rightarrow \mathcal{K}$ satisfies the inclusion (iii). Indeed, let $x, y \in K$ be fixed. Then

$$
\begin{aligned}
\alpha H(x)+\beta H(y) & =\alpha\left(\alpha F_{0}\left(\frac{x}{p}\right)+\beta F_{0}\left(\frac{y}{p}\right)\right) \\
& \subset \alpha F_{0}\left(\frac{p x+q y}{p}\right)=H(p x+q y) .
\end{aligned}
$$

Thus, by the minimality of $F_{0}, F_{0}(x)=H(x), x \in K$; i.e.,

$$
F_{0}(x)=\alpha F_{0}\left(\frac{x}{p}\right), \quad x \in K .
$$


Similarly we get

$$
F_{0}(y)=\beta F_{0}\left(\frac{y}{q}\right), \quad y \in K
$$

Hence, by (iii),

$$
F_{0}(x)+F_{0}(y)=\alpha F_{0}\left(\frac{x}{p}\right)+\beta F_{0}\left(\frac{y}{q}\right) \subset F_{0}(x+y)
$$

for every $x, y \in K$; i.e., $F_{0}$ is superadditive. In particular,

$$
n F_{0}\left(\frac{x}{n}\right) \subset F_{0}(x), \quad x \in K, n \in \mathbb{N} .
$$

Fix now $n \in \mathbb{N}$ and consider the s.v. function

$$
K \ni x \mapsto n F_{0}\left(\frac{x}{n}\right) \in \mathcal{K} .
$$

We have

$$
n F_{0}\left(\frac{x}{n}\right) \subset F_{0}(x) \subset F(x), \quad x \in K,
$$

$0 \in n F_{0}(0)$ and

$$
\alpha n F_{0}\left(\frac{x}{n}\right)+\beta n F_{0}\left(\frac{y}{n}\right) \subset n F_{0}\left(\frac{p x+q y}{n}\right), \quad x, y \in K .
$$

Therefore,

$$
n F_{0}\left(\frac{x}{n}\right)=F_{0}(x), \quad x \in K,
$$

by the minimality of $F_{0}$. Consequently,

$$
F_{0}(\lambda x)=\lambda F_{0}(x), \quad x \in K, \lambda \in \mathbb{Q}_{+} .
$$

Remark 4.3. Let $(X,\|\cdot\|)$ be a real normed space and $K \subset X$ a cone. Assume that $f: K \rightarrow \mathbb{R}$ is $\mathbb{Q}_{+}$-homogeneous. The function $f$ maps bounded subsets of $K$ onto bounded sets if and only if there exists a positive constant $M$ such that

$$
|f(x)| \leq M\|x\|, \quad x \in K
$$

Proof. Assume that $f: K \rightarrow \mathbb{R}$ is $\mathbb{Q}_{+}$-homogeneous (in particular, $f(0)=0$ ) and maps bounded subsets of $K$ onto bounded sets. Let

$$
M=\sup \{|f(x)|: x \in K \wedge\|x\| \leq 1\} .
$$

Fix $x \in K \backslash\{0\}$. There exists a decreasing sequence $\left(\lambda_{n}\right)_{n}$ such that

$$
\lim _{n \rightarrow \infty} \lambda_{n}=1 \quad \text { and } \quad \lambda_{n}\|x\| \in \mathbb{Q}_{+}, \quad n \in \mathbb{N} .
$$

Since

we have

$$
\left\|\frac{x}{\lambda_{n}\|x\|}\right\|=\frac{1}{\lambda_{n}}<1, \quad n \in \mathbb{N}
$$

$$
\left|f\left(\frac{x}{\lambda_{n}\|x\|}\right)\right| \leq M, \quad n \in \mathbb{N} .
$$

Consequently $|f(x)| \leq M\|x\|$.

The opposite implication is obvious. 
Theorem 4.4. Let $p, q>0, \alpha, \beta \in \mathbb{R}$ and $|\alpha|<p$. Assume that $(X,\|\cdot\|)$ and $(Y,\|\cdot\|)$ are real normed spaces, $K$ is a convex cone in $X$ and $F: K \rightarrow \mathrm{c}(Y)$ an s.v. function such that $0 \in F(0)$ and

$$
\alpha F(x)+\beta F(y) \subset F(p x+q y), \quad x, y \in K .
$$

If $K \ni x \mapsto \operatorname{diam} F(x) \in \mathbb{R}$ maps bounded sets onto bounded sets, then there exists a unique selection $f: K \rightarrow Y$ of $F$ fulfilling the equation

$$
\alpha f(x)+\beta f(y)=f(p x+q y), \quad x, y \in K .
$$

The selection $f$ is additive.

Proof. The family c $(Y)$ is closed under intersections of chains and

$$
\lambda \mathrm{c}(Y) \subset \mathrm{c}(Y), \quad \lambda \in \mathbb{R} .
$$

By Lemma 4.1, there exists a minimal s.v. function $F_{0}: K \rightarrow \mathrm{c}(Y)$ such that $F_{0}(x) \subset F(x), x \in K, 0 \in F_{0}(0)$ and

$$
\alpha F_{0}(x)+\beta F_{0}(y) \subset F_{0}(p x+q y), \quad x, y \in K .
$$

Lemma 4.2 shows now that $F_{0}$ is superadditive and $\mathbb{Q}_{+}$-homogeneous. Since $\operatorname{diam} F_{0}(x) \leq \operatorname{diam} F(x), x \in K$, the function $K \ni x \mapsto \operatorname{diam} F_{0}(x) \in \mathbb{R}$ maps bounded sets onto bounded sets and it is $\mathbb{Q}_{+}$-homogeneous. Hence, by Remark 4.3 , there exists a positive constant $M$ such that

$$
\operatorname{diam} F_{0}(x) \leq M\|x\|, \quad x \in K .
$$

Consequently, by Theorem 2.1, the s.v. function $F_{0}$ admits a unique selection $f_{0}: K \rightarrow Y$ such that

$$
\alpha f_{0}(x)+\beta f_{0}(y)=f_{0}(p x+q y), \quad x, y \in K .
$$

Since $f_{0}(0)=0$ and $f_{0}$ satisfies the above equation, $f_{0}$ must be additive. Assume that $f_{1}, f_{2}: K \rightarrow Y$ are selections of $F$ such that

$$
\alpha f_{i}(x)+\beta f_{i}(y)=f_{i}(p x+q y), \quad x, y \in K, i=1,2 .
$$

They are additive and

$$
f_{i}(x)=\alpha f_{i}\left(\frac{x}{p}\right), \quad x \in K, i=1,2 .
$$

Hence, for every $n \in \mathbb{N}$,

$$
f_{i}(x)=\alpha^{n} f_{i}\left(\frac{x}{p^{n}}\right), \quad x \in K, i=1,2 .
$$

Fix $x \in K$ arbitrary. Since $K \ni z \mapsto \operatorname{diam} F(z) \in \mathbb{R}$ maps bounded sets onto bounded sets, there exists a constant $M$ such that

$$
\operatorname{diam} F(y) \leq M, \quad\|y\| \leq 2\|x\| .
$$


For every $n \in \mathbb{N}$ there exists a number $q_{n} \in(1,2)$ such that $q_{n} p^{n} \in \mathbb{Q}_{+}$. Obviously, $\left\|q_{n} x\right\| \leq 2\|x\|, n \in \mathbb{N}$. Hence, for every $n \in \mathbb{N}$,

$$
\begin{aligned}
\left\|f_{1}(x)-f_{2}(x)\right\| & =|\alpha|^{n}\left\|f_{1}\left(\frac{x}{p^{n}}\right)-f_{2}\left(\frac{x}{p^{n}}\right)\right\| \\
& =|\alpha|^{n}\left\|f_{1}\left(\frac{q_{n} x}{q_{n} p^{n}}\right)-f_{2}\left(\frac{q_{n} x}{q_{n} p^{n}}\right)\right\| \\
& =\frac{1}{q_{n}}\left(\frac{|\alpha|}{p}\right)^{n}\left\|f_{1}\left(q_{n} x\right)-f_{2}\left(q_{n} x\right)\right\| \\
& \leq\left(\frac{|\alpha|}{p}\right)^{n} \operatorname{diam} F\left(q_{n} x\right) \leq\left(\frac{|\alpha|}{p}\right)^{n} M .
\end{aligned}
$$

Letting $n \rightarrow+\infty$ ends the proof.

\section{References}

[1] C. Badea, The general linear equation is stable. Nonlinear Funct. Anal. Appl. 10 (2005), 155-164.

[2] J. Brzdȩk and A. Pietrzyk, A note on stability of the general linear equation. Aequationes Math. 75 (2008), 267-270.

[3] J. Brzdȩk, D. Popa and B. Xu, Selections of the set-valued maps satisfying a linear inclusions in single variable via Hyers-Ulam stability. Nonlinear Anal. 74 (2011), 324-330.

[4] Z. Gajda, On stability of additive mappings. Int. J. Math. Math. Sci. 14 (1991), 431-434.

[5] Z. Gajda and R. Ger, Subadditive multifunctions and Hyers-Ulam stability. Numer. Math. 80 (1987), 281-291.

[6] D. R. Hyers, G. Isac and Th. M. Rassias, Stability of Functional Equations in Several Variables. Birkhäuser Boston, Boston, 1998.

[7] D. Inoan and D. Popa, On selections of generalized convex set-valued maps. Aequationes Math. 88 (2014), 267-276.

[8] S. M. Jung, Hyers-Ulam-Rassias Stability of Functional Equations in Nonlinear Analysis. Springer Optimization and Its Applications 48, Springer, New York, 2011.

[9] K. Nikodem and D. Popa, On selections of general linear inclusions. Publ. Math. Debrecen 75 (2009), 239-349.

[10] M. Piszczek, On selections of set-valued inclusions in a single variable with applications to several variables. Results Math. 64 (2013), 1-12.

[11] D. Popa, Additive selections of $(\alpha, \beta)$-subadditive set valued maps. Glas. Mat. Ser. III 36 (2001), 11-16.

[12] D. Popa, A stability result for general linear inclusion. Nonlinear Funct. Anal. App. 3 (2004), 405-414.

[13] D. Popa, A property of a functional inclusion connected with Hyers-Ulam stability. J. Math. Inequal. 4 (2009), 591-598. 
[14] A. Smajdor and J. Szczawińska, Selections of generalized convex set-valued functions with bounded diameter. Fixed Point Theory, to appear.

\author{
Andrzej Smajdor \\ Institute of Mathematics \\ Pedagogical University \\ Podchorążych 2 \\ PL-30-084 Kraków \\ Poland \\ e-mail: asmajdor@up.krakow.pl \\ Joanna Szczawińska \\ Institute of Mathematics \\ Pedagogical University \\ Podchorążych 2 \\ PL-30-084 Kraków \\ Poland \\ e-mail: jszczaw@up.krakow.pl
}

Open Access This article is distributed under the terms of the Creative Commons Attribution 4.0 International License (http://creativecommons.org/licenses/by/4.0/), which permits unrestricted use, distribution, and reproduction in any medium, provided you give appropriate credit to the original author(s) and the source, provide a link to the Creative Commons license, and indicate if changes were made. 